Ich eile nun zum Schlusse mit dem Bemerken, daß ich Ihnen in vorliegendem nur teilweise einen Einblick geben konnte in das Bemühen, die Frage des Saccharosenachweises in der Weise zu lösen, daß es auf einfachem Wege möglich wird, auch kleinste Mengen dieser für die Beurteilung mancher Waren wichtigen Zuckerart mit Sicherheit zuerkennen. Insoweit das noch nicht in vollem Maße gelungen ist, wie z. B. bei manchen Sübweinen, die ja auch in anderer Beziehung ein Sorgenkind unseres Berufs sind, werde ich mich bemühen, den letzten Stein noch zu entfernen, um glatten Weg zu schaffen.

\title{
Über Bonillonwïrfel.
}

\author{
Von
}

L. Geret in Antwerpen.

[Eingegangen am 14. September 1912.]

Die Bouillonwürfel sind eines der neuesten Erzeugnisse der Nahrungsmittelindustrie; daher ist auch noch wenig über ihre Zusammensetzung bekannt. Ebensowenig sind sie in Handbücher und die Ende des vorigen Jahrhunderts erschienenen "Vereinbarungen zur einheitlichen Untersuchung und Beurteilung von Nahrungs- und Genußmitteln" aufgenommen. Dagegen enthält der neu erschienene Codex alimentarius a ustriacus $(2,347)$ unter der Rubrik „Fleischextrakt und ähnliche Präparate, Abteilung c „Bouillonpräparate“, folgende Definition:

„Bouillonkapseln, Bouillonw ürfel, Bouillontafeln und ähnliche Präparate sind Gemische von Fleischextrakt mit Kochsalz, Fett, Gemüseauszügen und sonstigen Würzen. Sie dienen zur schnelleren Herstellung gebrauchsfertiger Fleischbrühe. Der Fleischextrakt ist als ihr wesentlicher Bestandteil aufzufassen".

Nachdem im Laufe der Zeit in der Bouillonwürfelfabrikation eine Reihe von Mißständen, darunter starke Preisunterbietungen und als unausbleibliche Folge dieses Konkurrenzkampfes Verschlechterung der Qualität, eingetreten war, erschien nach einem diese Kalamität schildernden Artikel in der Deutschen Nahrungsmittelzeitung ${ }^{1}$ ) und einem gleichlautenden in der Konservenzeitung ${ }^{2}$ ) eine ausführliche Arbeit von H. Sudendorfa) aus dem Staatlichen Hygienischen Institut zu Hamburg in dieser Zeitschrift, betitelt: "Welche Anforderungen sind an Bouillonwürfel zu stellen?" Sudendorf kommt nach Untersuchung einer größeren Anzahl in Hamburg vertriebener Bouillonwürfel zu folgenden sehr bemerkenswerten Schlüssen:

"Will man in dieser Frage den berechtigten Wünschen des Verbrauchers tunlichst Rechnung tragen und anch den Fabrikanten einen Spielraum zugestehen, so möge diese Grenze die Höchstgrenze für den Gehalt an Kochsalz - mit $65 \%$ in Vorschlag gebracht werden." . . .

„Bei dem Wirrwarr der im Handel unter ganz ähnlichen Bezeichnungen vorkommenden Präparate wie Suppenextrakt, Suppenwürze, Suppenwürfel u. a. ist weiterhin die Forderung zu stellen, dafi die Bezeichnung Bouillonwürfel nursolchenProdukten

1) Deutsche Nahrungsmittel-Zeitung No. 5 vom 1. März 1912.

2) Konserven-Zeitang vom 22. März 1912.

3) Diese Zeitschrift 1912, 28, 577. 
zaerkannt werden darf, deren kochsalzfreie Trockensubstanz im wesentlichen a as Fleischextrakt besteht. Daneben geringe Zusätze von aromatisierenden Bestandteilen zuzulassen, dürfte sich mit dieser Forderung wohl in Einklang bringen lassen."

„Keineswegs sollte aber geduldet werden, daß Fabrikate, deren kochsalzfreie Trockensubstanz ganz oder zum größten Teil aus Pflanzenauszügen, Hefenextrakt $u$. a. besteht, als Bouillonwürfel schlechthin feilgehalten werden. Solchen Präparaten muf der Deklarationszwang als Surrogat auferlegt werden, um zu verhindern, daß in Abnehmerkreisen der ursprüngliche Begriff Boaillonwürfel verwischt wird."

Sudendorf regt schließlich noch an, ,auch die Zusammensetzung der anderenorts gangbaren Marken festzustellen".

In der Arbeit Sudendorfs, die eine Orientierung und Anregung sein soll, ist außer Wasser, Asche, Kochsalz und Fett nur noch die Stickstoff-Substanz der Bouillonwürfel bestimmt worden.

Ich besitze nun eine große Anzahl Analysen (über 100) der verschiedensten Marken, die schon im Laboratorium der Liebig-Gesellschaft untersucht worden sind, und somit auch Erfahrung in dieser Sache. Das Ergebnis dieser Arbeiten im einzelnen zu veröffentlichen, hat hier wenig Zweck. Ich will nur zusammenfassend darauf hinweisen, daß der Gehalt der verschiedenen Würfeln an Fleischextraktivstoffen (worunter ich die im Liebig'schen Fleischextrakt enthaltene Menge Trockensubstanz, also $4 / 5$ des Extraktes verstehe) von etwa $20-25 \%$ in den besseren Marken bis auf 0 herab schwankt. Ich denke, nachdem ein staatliches Institut die dankenswerte Inititative zu einer Klärung und wenn möglich Normierung der Zusammensetzung der Bouillonwürfel ergriffen hat, die weitere Untersuchung und Urteilsbildung auch fernerhin diesen Instituten zu überlassen und möchte heute nur aus meiner Erfahrung zur Untersuchung der Bouillonwürfel auf ihren Gehalt an Fleischextraktivstoffen einige Anregungen für die Auswahl der grundlegenden Analysen machen, also lediglich Angaben, die von jedermann nachgeprüft werden können, deren Beachtung aber die Urteilsbildung fördern wird.

Man kann sagen, dab die Bouillonwürfel in runden Zahlen aus etwa $70 \%$ Wasser, Fett und Kochsalz und $30 \%$ Fleischextrakt und Gemüseextrakt und Würze oder zwei oder einem dieser Stoffe bestehen.

Fleisch- und Gemüseextrakte sind durch ihre Bezeichnung schon gekennzeichnet.

„Würze" ist ein Produkt aus Eiweißstoffen oder eiweißreichen Materialien, meist hergestellt durch Erhitzen mit starker Salzsäure bis zum Verschwinden der Biuretreaktion und nachfolgender genauer Neutralisierung durch Soda oder Ätznatron. Sie ist daher in der Hauptsache ein Gemisch von Kochsalz und Aminosäuren, besitzt den bekannten Suppenwürzengeschmack und ist natürlich viel weniger wertvoll als die Fleisch- und Gemüseextrakie.

Die Feststellung eines hohen Stickstoffgehaltes allein kann daher für die Bewertung eines Bouillonwürfels nicht dienen, weil gerade die billigen Würzen sehr stickstoffreich sind. Auch der Gehalt an kochsalzfreier Asche ist wenig brauchbar, weil auch Pflanzenauszüge aschereich sind. Das gleiche gilt, wenn schon in geringerem Grade, für den Gehalt an Phosphorsäure.

Als bestes Mittel zur Berechnung der Fleischextraktivstoffe erscheint mir der Gehalt an Gesamt-Kreatinin, der allerdings in abnormen Fällen noch durch eine Reihe anderer Bestimmungen kontrolliert werden muß, der aber normalerweise 
und in geübten Händen sehr zuverlässig ist. Die Gesamt-Kreatininmenge wird erhalten, wenn eine bestimmte Menge einer fettfreien Würfelmasselösung mit Salzsäure zur 'Trockene verdampft und das dabei in Kreatinin verwandelte Kreatin einschlieblich des bereits vorhandenen Kreatinins mittels der Folin'schen Methode quantitativ bestimmt wird.

Die der Folin'schen Methode zugrunde liegende Reaktion wurde bekanntlich von Jaffé ${ }^{1}$ ) entdeckt und besteht darin, daß Kreatinin mit gesättigter Pikrinsäure in alkalischer Lösung eine prachtvoll granatrote Färbung gibt. Sie wurde von Folin ${ }^{2}$ ) dadurch zu einer quantitativen Methode ausgearbeitet, daß er eine Lösung des Reaktionsgemisches von bestimmter Konzentration bezw. Färbung mit einer qualitativ gleich gefärbten 1/2 N.-Kaliumbichromatlösung verglich. Die Methode diente zunächst hauptsächlich zu physiologischen Untersuchungen und wurde schon in der verschiedensten Weise abgeändert, dabei teils verschlechtert, teils verbessert.

Zunächst seien aus der klassischen Arbeit Folin's die Hauptergebnisse angeführt :

$10 \mathrm{mg}$ Kreatinin, in $10 \mathrm{ccm}$ Wasser gelöst, geben die maximale Rotfärbung 5-10 Minuten nach Zusatz von $15 \mathrm{ccm}$ gesättigter, 1,2\%-iger Pikrinsäurelösung und $4-8 \mathrm{~cm} 10^{\circ} / 0$-iger Natronlauge. Die in dieser Weise erhaltene Lösung, auf $500 \mathrm{ccm}$ verdünnt, gibt eine Flüssigkeit, von der $8,1 \mathrm{~mm}$ in durchfallendem Licht genau dieselbe Farbe haben wie $8 \mathrm{~mm} 1 / 2$ N.-Kaliumbichromatlösung. Die Stärke der Färbung ist nach Erreichung des Maximums während der ersten 10 Minuten unverändert, schon nach einer halben Stunde ist sie aber merklich abgeschwächt.

Die zur Anwendung kommende Menge Substanz soll 7-15 mg Kreatinin enthalten, welche einschließlich Reagenzien auf $500 \mathrm{ccm}$ verdünnt werden; die Ablesung soll also Werte zwischen 5 und $13 \mathrm{~mm}$ ergeben. Noch sicherer ist es nach meiner Erfahrung, wenn man nur Werte von 6-10 mm gelten läßt, und wichtig ferner, daß auch die Menge der Reagenzien stets in annähernd gleichem Verhältnis zur Menge des Kreatinin und dem Volumen der Verdünnung steht. Man darf also nicht zu starke Lösungen einfach stärker verdünnen oder voraussichtlich schwache konzentrierter lassen.

Nach der Arbeit Folin's kamen ausführliche Versuche zum Studium des Kreatinstoffwechsels durch van Hoogenhuyze und Veeploegh ${ }^{3}$ ) sowie durch Gottlieb und Stangassinger ${ }^{4}$ ). Diese Arbeiten lehnten sich im analytischen 'Teil eng an die Vorschriften Folin's an, benutzten aber statt des teuren Dubosc q'* schen Colorimeters viel billigere eigener Konstruktion.

Van Hoogenhuyze und Veeploegh fanden u. a., daß die Temperatur der Kreatininpikratiösung einen Einfluß auf die Färbung hat und zwar die Farbintensität proportional zur Temperatursteigerung zunimmt. Man soll also zum Verdünnen auf $500 \mathrm{ccm}$ möglichst Wasser von mittlerer Temperatur nehmen.

Die Jaffé-Folin'sche Methode gibt bekanntlich nur den Kreatiningehalt an; Kreatin reagiert nicht auf Pikrinsäure. Um das Kreatin mit zu bestimmen, muß es durch Erhitzen mit Säure in Kreatinin umgewandelt werden (schwaches Alkali, z. B.

\footnotetext{
1) Zeitschr. physiol. Chem. 1886, 10, 399.

$\left.{ }^{2}\right)$ Zeitschr. physiol. Chem. 1904, 41, 233.

$\left.{ }^{3}\right)$ Zeitschr. physiol. Chem. 1905, 46, 415.

4) Zeitschr. physiol. Chem. 1907, 52, 1.
} 
Kalkmilch, wandelt wieder Kreatinin in Kreatin zurück). Außer Ja ffé ${ }^{1}$ ) haben besonders Gottlieb und Stangassinger diesbezügliche Untersuchungen angestellt und gefunden, da die quantitative Umsetzung von Kreatin in Kreatinin erfolgen kann durch 4,32\% $\%$-ige Schwefelsäure bei $100^{\circ}$ (in kochendem Wasserbade) innerhalb $2^{1 / 2}$ Stunden und durch 4,56\%-ige Salzsäure auf dem kochenden Wasserbade in 2 Stunden.

Bei geringerer Säurekonzentration und bei einfachem Abdampfen mit gleichverdünnter Säure wurde weniger erhalten. Mit konzentrierterer Säure und ganz besonders beim Erhitzen unter Druck wurde nach Gottlieb und Stangassinger sowie nach Jaffé erheblich weniger gefunden; es kann also auch Kreatinin zerstört werden.

Die ersten, welche die Methode dann zur Untersuchung von Fleischextrakt anwendeten, waren Baur und Barschall2). Diese Forscher fanden:

1. daß bei der außerordentlichen Empfindlichkeit des Verfabrens und der dadurch bedingten Untersuchung in sehr verdünnter Lösung die geringe Färbung der Fleischextrakte nicht in Betracht kommt,

2. daß keine Störung durch irgend einen der zahlreichen mitanwesenden Stoffe bewirkt wird,

3. daß die Überführung des Kreatins in Kreatinin durch Erwärmen der zu untersuchenden Lösung auf dem Wasserbade in $1 / 3$-normaler (1,2\%-iger) salzsaurer Lösung während 4 Stunden vollzogen werden soll.

Sie benutzten ein Colorimeter von Duboscq.

Die von ihnen gefundenen Werte für Liebig's Fleischextrakt, 3,0\% Kreatinin $+1,75 \%$ Kreatin oder 3,95\% sogenanntes Gesamt-Kreatinin (d. h. nach der Inversion durch Säure gefundenes Kreatinin, also Kreatinin + invertiertes Kreatin) sind viel zu niedrig, da nach verbesserten Methoden in Standardextrakt stets mindestens etwa $6 \%$, meist darüber an Gesamt-Kreatinin gefunden wird. Der Fehler ist

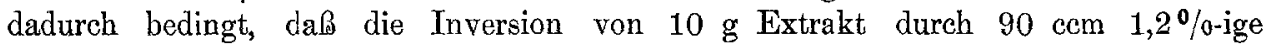
Salzsäure unvollständig ist und die auch von Baur und Barschall geübte Verdünnung der invertierten Lösung auf das Drei- und Vierfache von $500 \mathrm{ccm}$ unrichtige Ergebnisse bei der Ablesung liefert, weil dann der Pikrinsäurezusatz verhältnismäßig zu gering ist.

Von nordamerikanischen Forschern beschäftigte sich besonders Grindley in Verbindung mit $\mathrm{Woods}^{3}$ ) mit der neuen Methode und untersuchte kalt hergestellte Fleischauszüge, in denen er kein Kreatinin sondern nur Kreatin fand, sowie auch einige Fleischextrakte, in denen er 1,30 bis 6,38\% Gesamt-Kreatinin fand. Die Inversion nahm er nach Zusatz von $1 / 10$ N.-Salzsäure durch wiederholtes Eindampfen der Extraktlösungen auf ein kleines Volumen vor.

Unterdessen hatte Folin in der Festschrift für Hamarsten (1906) seine Inversionsmethode durch Verwendung stärkerer (Normal-) Salzsäure verbessert und dafür die Menge der zur Farbenreaktion verwendeten Natronlauge von $5 \mathrm{ccm}$ auf 9 cem erhöht.

1) Zeitschr. physiol. Chem. 1906, 48, 434.

2) Arbeiten aus dem Kaiserl. Gesundheitsamte 1906, 24, 552.

3) Journal of Biolog. Chem. 1907, 2, 309. 
Ebenso hatten Benedikt und Meyers ${ }^{1}$ ) die Inversion in $1 / 2$ N.-Salzsäure durch 30 Minuten langes Erhitzen im Autoklaven bei $117-120^{\circ} \mathrm{C}$ mit gutem Erfolg versucht und empfohlen.

Dann trat noch $\mathrm{O}$. Hehner mit Abänderungen auf ${ }^{2}$ ), die von der Voraussetzung ausgingen, daß die Reaktion auf der Bildung von Kreatininpikrat beruht, welches in Natronlauge mit blutroter Farbe löslich jst; er ist der Ansicht, daß Baur und Bar s chall, sowie Grindley zu wenig Gesamt-Kreatinin fanden, weil sie nicht genug Pikrinsäure zugesetzt hätten. Er fand in Liebig's Fleischextrakt und einigen anderen Extrakten 10-12\% Gesamt-Kreatinin; ferner stellte er fest, daß ein Überschuß an Alkali das Ergebnis an Kreatinin vermindert. Seine Theorie und seine Ergebnisse wurden durch eine Erklärung von Chapman, Dyer und Salamon ${ }^{3}$ ) anerkannt.

Grindley aber prüfte in einer ausgezeichneten Arbeit ${ }^{4}$ ) seine früheren und Hehner's Angaben nach und kam zu dem Schlusse, daß Hehner, der im übrigen keinerlei genaue Angaben gemacht hatte,

1. gar keine Kaliumchromatlösung, sondern nur eine sogenannte StandardKreatininlösung zum Vergleich benutzt hatte;

2. daß er (Grindley) noch annähernd die gleichen Ergebnisse erhalte wie früher, keineswegs aber die enorm hohen Zahlen Hehner's;

3. daß aber der Befund ein wenig größer sei, wenn man $10 \mathrm{ccm}$ Alkalilösung statt $5 \mathrm{ccm}$ verwende;

4. daß der Befund bei der Bestimmung des fertiggebildeten Kreatinins nicht vergrößert werde durch Vermehrung der Pikrinsäuremenge (also es bei $15 \mathrm{ccm}$ bleibe); daß aber der Befund an Gesamt-Kreatinin größer werde, wenn $25 \mathrm{ccm}$ anstatt $15 \mathrm{ccm}$ Pikrinsäure angewendet werden;

5. "That the Folin method when properly modified, is as applicable to meat extracts and meats as it is to urine, and that it gives reliable and concordant. results in the hands of different analysts of this laboratory."

Er schlägt als Ergebnis seiner Untersuchungen vor,

1. für das präformierte Kreatinin, die etwa $10 \mathrm{mg}$ Kreatinin enthaltende Menge mit $15 \mathrm{ccm}$ Pikrinsäurelösung und $10 \mathrm{ccm}$ Natronlauge zu versetzen etc.;

2. für das sog. Gesamt-Kreatinin eine etwa $40 \mathrm{mg}$ Gesamt-Kreatinin enthaltende Menge einer Lösung im Becherglase auf $10 \mathrm{ccm}$ zu konzentrieren und, mit $10 \mathrm{ccm}$ N.-Salzsäure versetzt, im Autoklaven 30 Minuten bei $117-119^{\circ} \mathrm{C}$ zu behandeln, dann auf $100 \mathrm{ccm}$ zu verdünnen und $25 \mathrm{ccm}$ davon (= etwa $10 \mathrm{mg}$ GesamtKreatinin) mit $30 \mathrm{ccm}$ Pikrinsäurelösung und $10 \mathrm{~cm}$ Natronlauge wie gewöhnlich in Reaktion zu bringen.

Auf dem Internationalen Kongreß für angewandte Chemie in London hielt A. Ch. Chapmans einen Vortrag, in dem er zeigte, daß die Färbung nicht auf der Bildung von Kreatininpikrat, sondern auf einer Reduktion der Pikrinsäure durch das Kreatinin (zu Pikraminsäure etc.) beruhe und daß die sehr komplizierte Reaktion nur unter genau eingehaltenen Arbeitsbedingungen genaue Ergebnisse liefern

$\left.{ }^{1}\right)$ Americ. Journal of Physiol. 1907, 18, 398.

2) Pharmaceutical Journal 1907, Supplement 683.

3) Pharmaceutical Journal, 1907, Supplement 690.

\$) Journal of Biolog. Chemistry 1907, 8, 491 . 
könne. Er betonte nochmals die Wichtigkeit konstanter Temperatur (vergl. van Hoogenhuyze und Veeploegh), verneinte aber den Einfluß von gleichzeitig vorhandener Glykose (trotzdem die Reaktion auf Reduktion beruht).

An gleicher Stelle endlich sprach F. C. Cook, der nichts Neues brachte, als daß die Gegenwart von koagulierbarem Eiweiß, sowie Albumosen und Pepton den Kreatingehalt in Extrakten vermindere. Diesen Befund konnte ich bei Versuchen von verschiedenen Gemischen aus Standardextrakt und Leim nicht bestätigen, fand im Gegenteil aber eine ganz geringe Menge Gesamt-Kreatinin mehr in den Mischungen von 50\% Fleischextrakt $+50 \%$ Leim und $25 \%$ Fleischextrakt $+75 \%$ Leim, als die Berechnung ergab.

Meine Methode lehnt sich an die frühere von Grindley an.

Nach diesen Ausführungen bleiben mir noch folgende Bemerkungen zu machen:

Ein höherer Gesamt=Kreatiningehalt kann vorgetäuscht werden, wenn viel Caramel oder Zucker, der bei der Salzsäurebehandlung caramelisiert wird, zugegen ist. Auch in diesem Falle sind noch brauchbare Ergebnisse zu erhalten, wenn nach der Inversion mit geeigneter Tierkohle behandelt wird.

Es gibt Personen, welche infolge leichter Farbenblindheit die orangegelben Abstufungen im Colorimeter nicht genügend scharf erkennen. Diese sind durch Vergleich der von mehreren Personen (innerhalb 10 Minuten!) abgelesenen Werte zu ermitteln und eventuell beim Ablesen zu ersetzen.

Meine Zusammenstellung sollte zeigen, wie sehr schon bei geringer Abänderung der Methode die Ergebnisse abweichen können. Sie soll daher auch eine Anregung sein, daß sich jeder Untersucher, der die Methode benutzen will, erst erschöpfend durch Kontrollversuche einarbeitet und schließlich in seiner Veröffentlichung die Art der gewählten Methode genau beschreibt.

Bei richtiger Anwendung gibt die Folin'sche Methode aber sehr gleichmäbige und brauchbare Zahlen, und sie wird sicher dazu beitragen, die verschiedenen Bouillonpräparate auf ihren wahren Gehalt an Fleischextraktivstoffen, ihren ,wesentlichen Bestandteil “, zuverlässig zu prüfen.

\section{Über Samen und Samenöl der Heidel- und Preißelbeere.} Von

\section{A. Diedrichs.}

Mitteilung aus dem Staatlichen Chemigchen Untersuchungsamte für die Aaslandsfleischbeschau zu Goch.

[Eingegangen am 17. September 1912.]

\section{Heidelbeere.}

Die Heidelbeere, auch Wald-, Schwarz-, Blau- oder Bickbeere genannt - die Frucht von Vaccinium Myrtillus L., einer Ericacee - zählt zu den wichtigeren unserer einheimischen Beerenobstarten. Namentlich für die gebirgigen Gegenden sind diese Beeren von einer gewissen wirtschaftlichen Bedeutung, insofern ihr Sammeln eine nicht unbedeutende Einnahmequelle, besonders für die ärmere Gebirgsbevölkerung bildet. 